\title{
Do-It-Yourself Artificial Pancreas Systems in Type 1 Diabetes: Perspectives of Two Adult Users, a Caregiver and Three Physicians
}

\author{
Dominic C. Marshall · Melissa Holloway • Mendy Korer • \\ James Woodman · Anna Brackenridge · Sufyan Hussain
}

Received: July 1, 2019 / Published online: August 22, 2019

(C) The Author(s) 2019

\begin{abstract}
Advances in continuous glucose monitoring and insulin pumps have allowed people with type 1 diabetes (T1D) and caregivers to accurately and continuously measure their glucose levels and make adjustments to insulin infusion. In recent years, algorithms for subcutaneous insulin dosing have been developed that can respond to changes in glucose in an automated fashion and "close the loop". At present, a first-generation
\end{abstract}

Enhanced Digital Features To view enhanced digital features for this article go to https://doi.org/10.6084/ m9.figshare.9249995.

D. C. Marshall · A. Brackenridge $\cdot$ S. Hussain ( $\square)$ Department of Diabetes and Endocrinology, Guy's and St Thomas NHS Foundation Trust, London, UK e-mail: s.hussain@imperial.ac.uk

D. C. Marshall

Critical Care Research Group, Nuffield Department of Clinical Neurosciences, University of Oxford, Oxford, UK

M. Holloway

Speaking Diabetes Ltd, London, UK

M. Korer

London, UK

J. Woodman

Cambridge, UK

S. Hussain

Department of Diabetes, Endocrinology and

Metabolism, Imperial College London, London, UK 'hybrid closed-loop', 'artificial pancreas' or 'automated insulin dosing' system, Medtronic 670G, is available commercially. Further systems are in clinical trials. Frustrated by the slow pace of innovation, people affected by diabetes have united online under the hashtag '\#WeAreNotWaiting,' to disseminate opensource diabetes technologies. One dimension of \#WeAreNotWaiting is "looping" with a do-ityourself artificial pancreas. Here we provide the perspectives of two adults with T1D, the parent of a child with T1D and three physicians who detail their experience with these systems. These personal and clinical perspectives highlight very clear metabolic and psychological benefits of these systems in real-world settings.

Keywords: Artificial pancreas; Automated insulin dosing systems; Closed-loop insulin dosing; Do-it-yourself; Hybrid closed-loop insulin dosing; Looping; \#WeAreNotWaiting

\section{PERSPECTIVES FROM TWO ADULTS WITH T1D AND THE PARENT OF A CHILD WITH T1D}

\section{James's Story}

I have lived with type 1 diabetes since 2002 . The last year has seen a technological transformation 


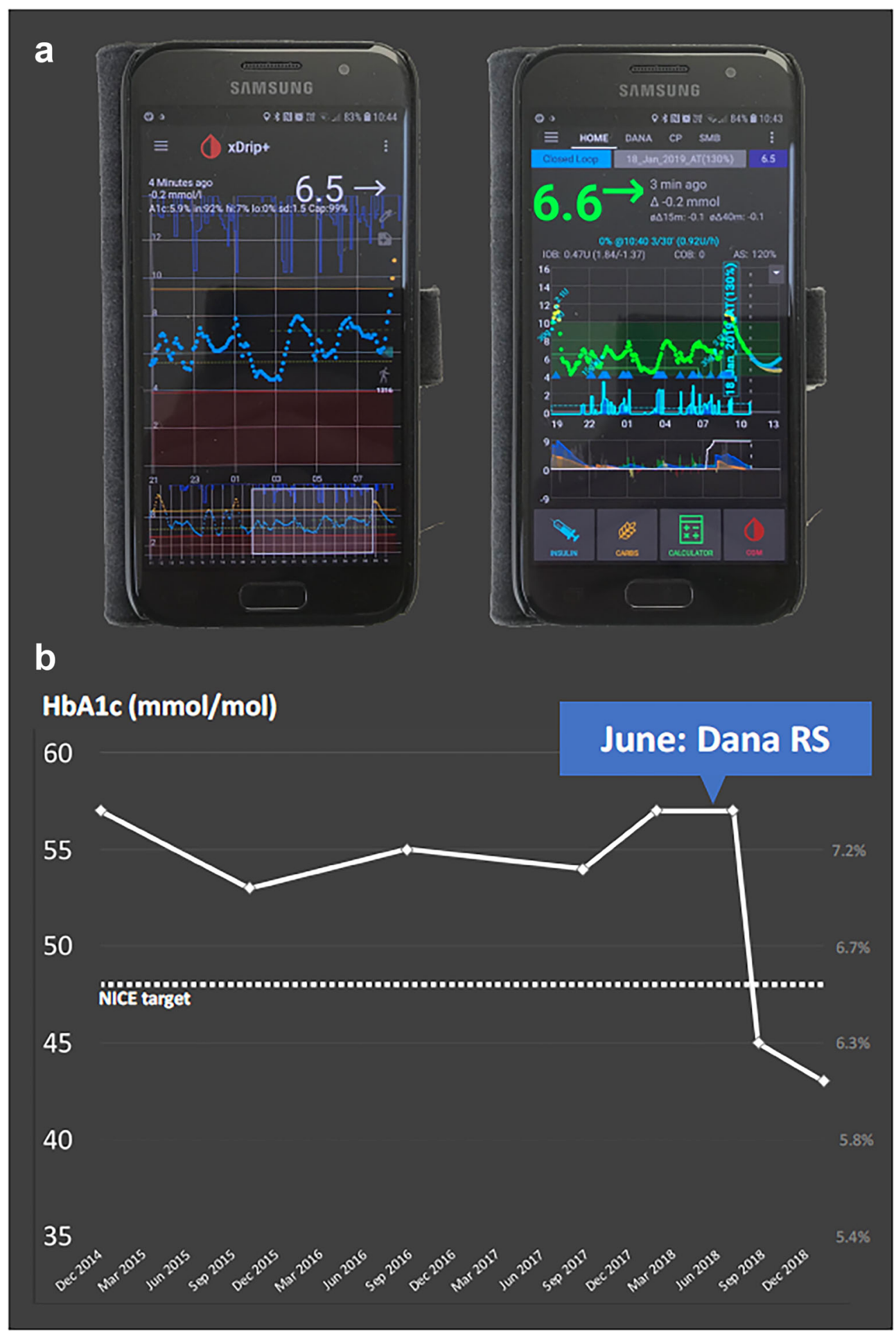

Fig. 1 a Smartphone screenshots of xDrip+ with CGM data and AndroidAPS app on an Android phone used by James Woodman. The AndroidAPS app uses the OpenAPS algorithm to calculate insulin dose adjustments from the CGM data. The screenshot illustrates the interface and display indicating insulin dose adjustments The Android phone is the controller and communicates via Bluetooth to a Bluetooth-enabled pump. b HbAlc reduction in response to use of DIY APS system from June 2018 for James Woodman 
in both measurable outcomes and quality of life. I'm not cured, but this is the next best thing for now.

The core tools I use to control the condition haven't changed. I have a conventional insulin pump funded by the NHS-the Dana RS. I selffund the Dexcom G6 continuous glucose monitoring system (CGM).

The life-changing shift was my decision to connect those two devices: to allow a mobile phone app to take control of my pump and to adjust insulin dosing in response to blood glucose data (Fig. 1a). The components form a closed loop system-sometimes known as an artificial pancreas. Users like me ('loopers') call it 'looping'.

Without a fully functioning pancreas, it's hard to match active insulin to the body's changing needs. That's why diabetes is toughand it's why most of us don't meet healthcare targets. An insulin pump gives greater flexibility, but it doesn't make the problem go away.

Looping changes that by predicting the future. If my blood glucose is falling, or if the system calculates that I'm going to become hypoglycaemic, it can reduce the supply of insulin in advance. If my blood glucose is rising, it can deliver more insulin-within strictly defined safety constraints. It's like a sliding scale where you rethink the dose every $5 \mathrm{~min}$.

Should I give a correction bolus? Should I change my basal rate? How can I reverse that trend? I used to invest lots of energy in answering questions like these. Now, the app handles them on my behalf. It's not perfect, but it does a much better job than me.

Before looping, my $\mathrm{HbA}_{1 \mathrm{c}}$ was around $57 \mathrm{mmol} / \mathrm{mol}$. I worked hard to bring it down, but failed, year after year. In June 2018 I handed over control to the artificial pancreas, and by August my $\mathrm{HbA}_{1 \mathrm{c}}$ had fallen to $45 \mathrm{mmol} / \mathrm{mol}$. By January 2019 it was $43 \mathrm{mmol} / \mathrm{mol}$ (Fig. 1b). I typically spend $85-90 \%$ of the day between 4 and $10 \mathrm{mmol} / \mathrm{L}$. The positive mental impact has been profound: I spend significantly less time managing diabetes, with improved results.

The system I use-AndroidAPS-is free to anyone with access to the Internet. Whilst the system is freely available, there are barriers. You need CGM and a compatible Bluetooth-enabled pump. You need to build and configure an app on an Android device. Even when you can see the potential benefits, it's easy to feel discouraged by technology. The good news is that you don't need to be a computer expert-and that the online community of loopers wants new users to succeed.

The features of AndroidAPS go beyond any commercial offering, and it is open-source: designed, maintained, supported and given away by a global community of volunteers. Nobody makes money from it or from two other similar systems, OpenAPS and Loop. No regulator has approved them either. This is the cutting edge of patient-led healthcare innovation, driven by people who don't want to wait for the medical device industry to deliver the next generation of technology. Search online for \#WeAreNotWaiting to learn more about this approach.

Is it risky? I have no adverse events to report. There may be unknown risks that come with DIY technology, but for me, looping reduces risk by improving both short- and long-term glycaemic control.

I realise that I am privileged, and part of my good luck is that I attend a great clinical service. I needed a specific pump to make looping possible, so access to that critical part of the system depended on my diabetes team. I explained what I wanted to do, why I believed it was right for me, and that I was not asking them to take responsibility for an unlicensed system. It took some negotiation, but they were interested and respectful from the start, and they did not erect unnecessary barriers.

That positive attitude extended to a recent hospital admission and cholecystectomy. The loop stayed active on the ward whilst I was too ill to care for myself, and the anaesthetist took the view that it was safe to let the system look after my blood glucose during surgery. I am convinced that AndroidAPS can take some of the credit for my fast recovery, and I am extremely grateful to everyone at the hospital for their willingness to accept my choices.

Loopers are still a tiny minority, but the number is growing. I believe that when patients choose to manage a condition like diabetes in ways that challenge the norm, we start to break 
down barriers. That can help to move healthcare technology forward for everyone.

\section{Mendy's Story}

In April 2017, our 11-month-old son was diagnosed with T1D at University College London Hospitals NHS Foundation Trust (UCLH). As our child was so young, we were fully funded to have a pump, Medtronic 640G, and the Enlite CGM sensors. Within the first week of diagnosis we had training on the pump, sensor and general management including carb counting. My son could be released from hospital only after the training was complete.

Six months later, this is what his diabetes management looked like:

- Rapid glycaemic fluctuations on a daily basis

- Daily hypoglycaemic events due to correctly calculated but still excessive insulin boluses

- Daily correction boluses to bring down glycaemic excursions

- Multiple overnight glucose checks by his mum and me

- Best $\mathrm{HbA}_{1 \mathrm{c}} 64 \mathrm{mmol} / \mathrm{mol}$

To our friends and family, he looked like a toddler with delayed development, and we looked like parents stressed due to lack of sleep. We were constantly anxious due to lack of control, we had to cut down our work hours, and weekly conversations with the paediatric endocrinologist and nurses for guidance had limited impact. We explored what medical systems were available in other developed countries and despaired: we already had the best possible tools, apparently.

In October 2017, our paediatric endocrinologist referred us into a closed-loop system trial. On day 2 of looping we saw the first ray of magic, during the night (with no food influencing sugar levels) we saw a steady, safe CGM line. The system was self-adjusting the basal insulin to maintain constant 'in range' levels. We could sleep, but more than being undisturbed for 7 hours, we could sleep anxiety-free. No worry if my child will be alive the next morning, not jumping out of bed due to a car alarm out on the street and thinking that was the pump's alarm, nothing; just sleep, for us and our child.

But we were on a 3-month study and after that we had to give back the pump and technology to the investigators.

In January 2018, after sourcing the hardware, some really kind tech people helping with software and a supportive doctor, we set up OpenAPS (Fig. 2a-c). Over the past ca. 16 months we have been constantly tweaking the system to improve ease of management.

After 1 year of looping:

- Daily average time in range (3.6-14 mmol/ L), $91 \%$

- Daily average carb intake, $128 \mathrm{~g}$

- Nighttime hypo treatments in an average month, 1 (one!)

- Both low and high glycaemic index foods all able to be managed safely with the system

- Best $\mathrm{HbA}_{1 \mathrm{c}}, 45.4 \mathrm{mmol}$

- Controlled, safe glucose levels during occasional illnesses

Whilst the condition is not cured, the ability to use an automated system for T1D management is like pressing autopilot on an uncontrolled aeroplane.

The biggest improvement for a toddler has to be flexibility in food management. As a parent, I cannot be sure how much he will eat at mealtimes, but the system compensates by identifying the glucose rise as food comes in. As a result, I can let my toddler be a toddler, without the need for micromanagement.

Finally, the system makes adjustments every 5 minutes, 24 hours a day, factoring in carb amounts, carb timing, glycaemic index, insulin on board, deviations, etc. It does more to manage my son's diabetes than I can possibly do as a devoted caregiver.

There are some concerns about using a nonlicensed product and we take absolute responsibility for this. Current T1D management includes avoiding hypos (the most immediate danger). What is the primary cause of hypo? Although almost always unintended, it is due to excess insulin. Every hypo treatment is because there is too much insulin in the body and the only way the insulin got inside is from a human miscalculating how much to give due to the 


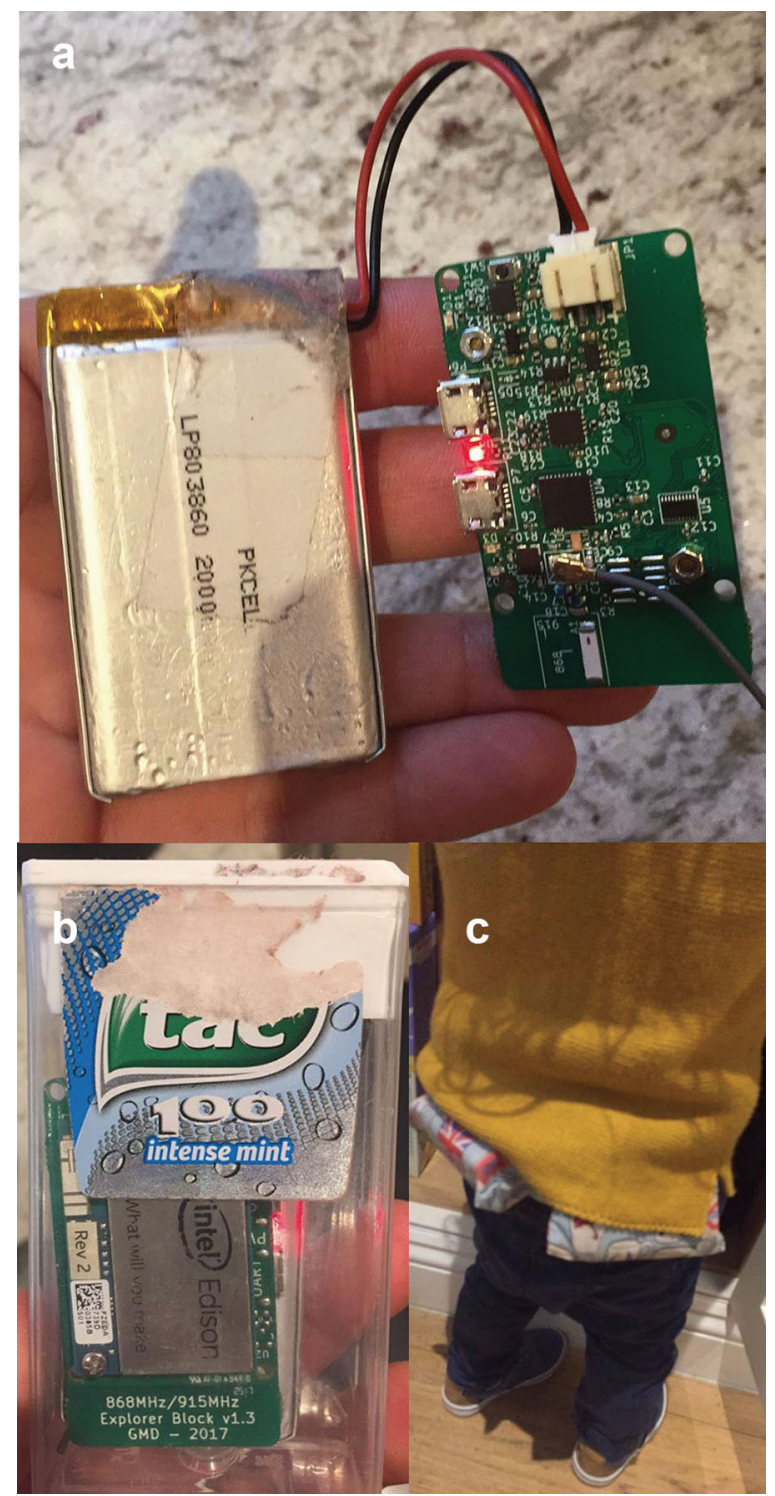

Fig. 2 a OpenAPS controller 'rig' built from an Edison explorer board. This 'rig' holds the OpenAPS algorithm and fcommunicates via radio to Mendy Korer's 3-year-old son's insulin pump and CGM. b OpenAPS controller 'rig' carried in its case built from a Tic Tac box. c Two carrying waist pouches worn by Mendy Korer's 3 year-old son. One of these contains the insulin pump and the other contains the 'rig' Edison Explorer board in its Tic Tac box case

multitude of variables in blood glucose levels. I've got a surprise solution-computers! If you can teach a computer all the rules and variables to micromanage glucose levels by giving small amounts of extra insulin (or reducing insulin), then recalculating every $5 \mathrm{~min}$, bingo, we have our solution: less overtreatment, fewer hypos.

\section{Melissa's Story}

I first became aware of the \#WeAreNotWaiting movement a few years ago via people who were into Nightscout, the open-source diabetes data visualization and remote monitoring system. I thought the first wave of people using DIY artificial pancreas systems showed impressive initiative, but didn't think I would ever join them. It sounded too complicated and techy, and I had great control using a pump (started 1996) and CGM (started 2006) as separate components anyway.

However, from autumn 2015, things changed. Following the birth of my first child in March that year, I was diagnosed with postpartum thyroiditis. Despite my thyroid hormone levels looking fine and stable on daily levothyroxine, after my thyroid quit my blood sugars fluctuated like never before. Having always used tubed pumps, in October 2017 I switched to OmniPod, thinking it would help me to be able to set temporary basal rates and deliver boluses more discreetly. Maybe that would help me feel more in control of my diabetes.

Despite an $\mathrm{HbA}_{1 \mathrm{c}}$ between 42 and $44 \mathrm{mmol} / \mathrm{mol}$, by early 2018 frequent dramatic glycaemic excursions made me think it would be unwise to try to have a second child. That thought was jarring to me because I had never let T1D tell me what to do. T1D has always been a variable I account for, never an excuse not to do something.

At the Advanced Technologies and Treatments for Diabetes (ATTD) conference in February 2018, I heard Aaron Kowalski, now the first person with T1D to serve as President and CEO of JDRF International, talk about his personal experience of DIY looping. I was fascinated and envious. I joined the Looped group on Facebook shortly after ATTD, thinking maybe I would attempt to 'open-loop' with OmniPod. In open looping, an independent algorithm running on a phone suggests basal rate adjustments for the user to action manually. That was too complicated for me to 
Week 2

Thu 31 May 2018 - Wed 6 Jun 2018

a Mon Tue Wed Thu Fri Sat Sun

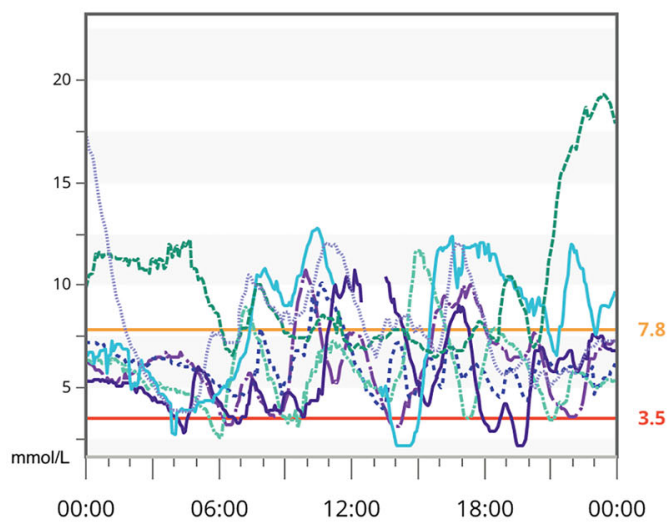

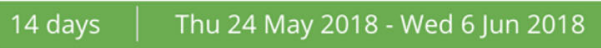

b

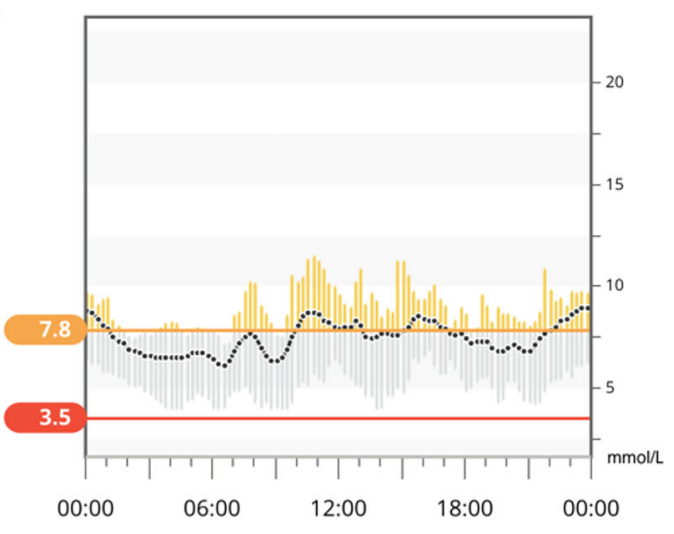

C

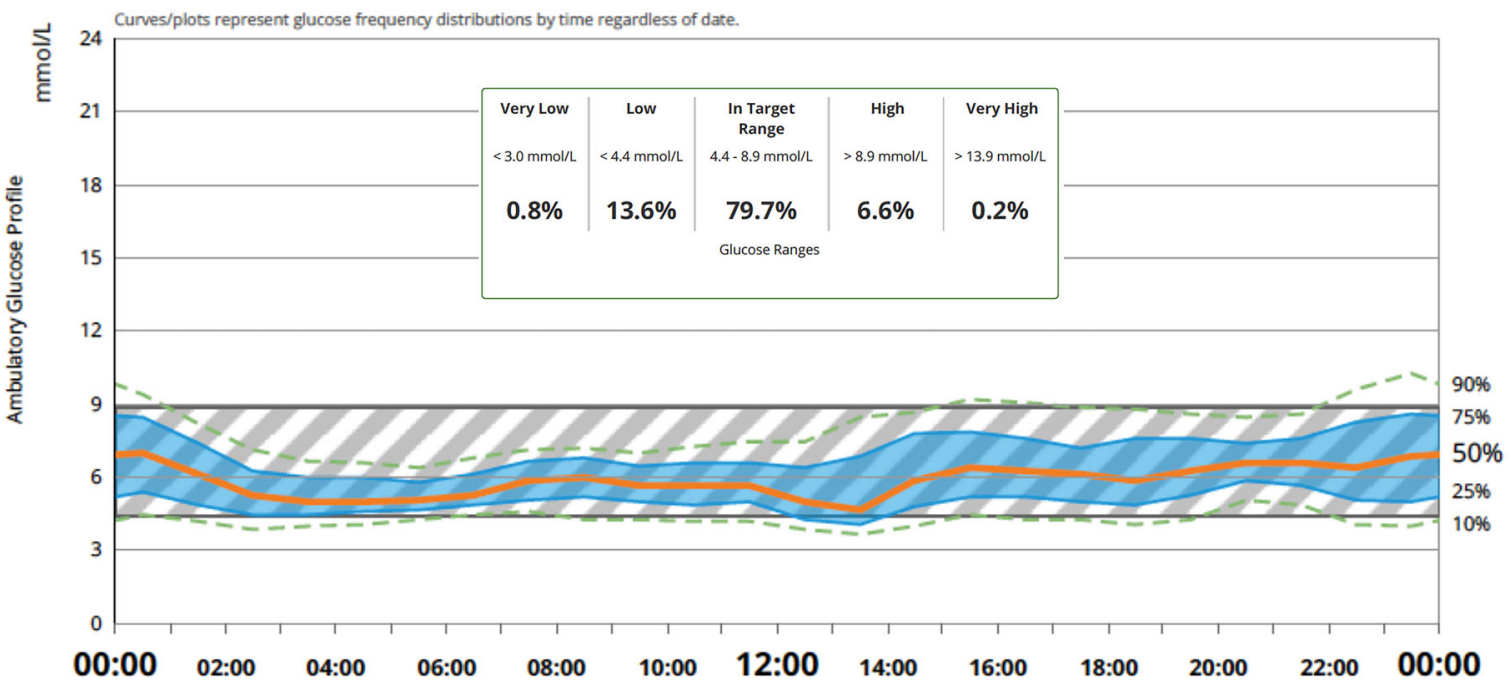

Week 2

Fri 31 May 2019 - Thu 6 Jun 2019

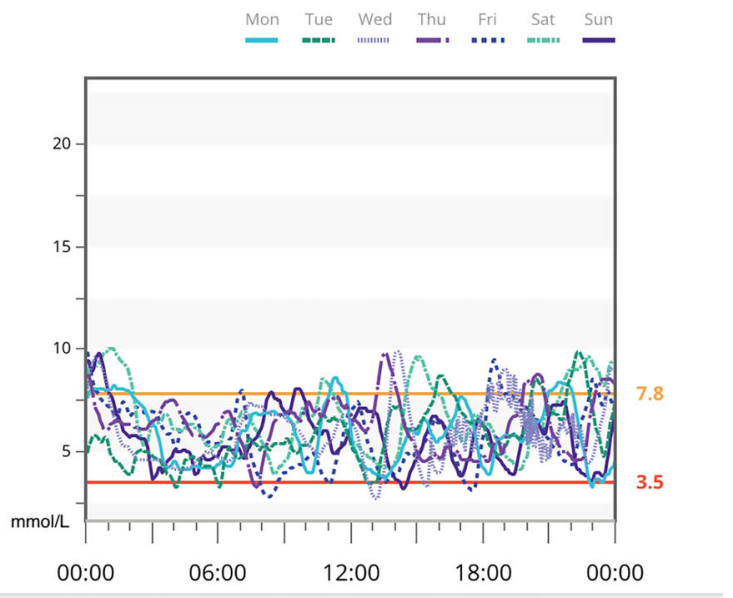

\section{4 days $\quad$ Fri 24 May 2019 - Thu 6 Jun 2019 ๑}

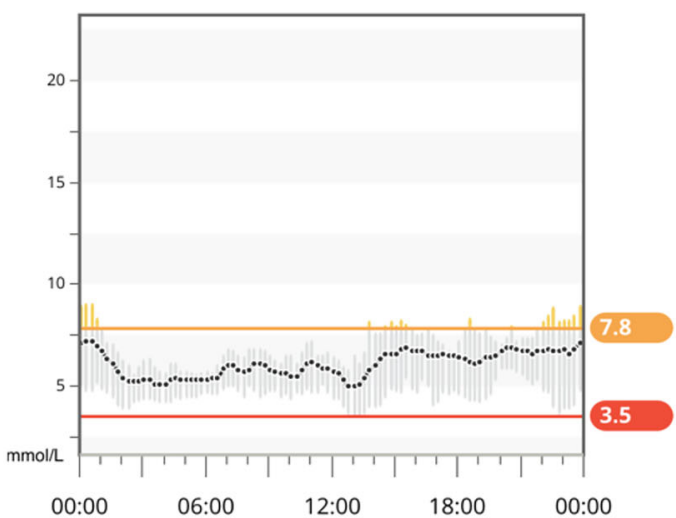


4Fig. 3 a A 7-day overlay modal day view CGM profile before (left) and after (right) use of DIY Loop APS system by Melissa Holloway during pregnancy demonstrating improvements in overnight control and daytime excursions with reduced inter- and intra-day glycaemic variability. b A 14-day trend view with mean (dotted line), range within time blocks (grey bars) and percentage time in range (3.5-7.8 mmol/L) before (left) and after (right) use of DIY Loop APS system by Melissa Holloway during pregnancy demonstrating significant improvement in time in range with minimal hypoglycaemia. c A 14-day ambulatory glucose profile using DIY Loop APS system by Melissa Holloway during pregnancy demonstrating low inter- and intra-day glycaemic variability

attempt, but by mid-June 2018, having learned a lot from Looped and Looped UK, I was gifted a secondhand loopable pump, I bought a RileyLink (\$150) and I built my Loop app (\$100 for an Apple Developer annual subscription). I already had a serviceable (if ageing) MacBook Pro to run the app build on. I have had NHS funding for Dexcom CGM for over 10 years due to hypoglycaemia unawareness, so the cost of CGM was not an issue for me.

I found the trickiest step was setting up Nightscout, because I misread a line of the instructions. After $20 \mathrm{~min}$ of frustration, I posted a request for advice in the Looped UK group and got help to resolve the issue within $15 \mathrm{~min}$. After a week of open-looping with my new setup, I closed the loop on 18 June 2018.

At my routine pump clinic appointment 2 weeks later, my consultant made it clear that I am using an old, out-of-warranty insulin pump at my own risk (fine with me because I have two spares!). She changed the purchase order for my pump consumables to enable me to get the infusion sets and reservoirs I need for looping.

What did I most hope for from using a DIY hybrid closed-loop? I wanted to have the kind of diabetes control I was used to before my first pregnancy, where $12 \mathrm{mmol} / \mathrm{L}$ was a big high and I didn't see it much. I wanted to sleep soundly through the night and wake up with my glucose levels in my target range, so I could start my day with a smile. I wanted to spend time with my son and not worry that I should be doing something about my blood sugar when he wanted to play. Most of all, I wanted to feel like it would be safe to try to have another baby. The only fear I had was that I would not get the results I was looking for.

After 3 months of looping, I felt like things were going really well. My husband and I started trying for a baby. By December 2018, we both felt confident that my diabetes control was less of an issue than my age (37.5). This past January we went on holiday for 10 days. As of the time of writing (19 June 2019) I'm 24 weeks pregnant.

Starting from the day I found out I was pregnant, my time in range for 3.5-7.8 $\mathrm{mmol} / \mathrm{L}$ (pregnancy closed-loop target range) is $80 \%$ (vs. the goal of $70 \%$ ) and my median high glucose level is $8.9 \mathrm{mmol} / \mathrm{L}(\mathrm{SD} \pm 1.0)$. My mean glucose within my target range is $5.5 \mathrm{mmol} / \mathrm{L}$. The difference in my diabetes control from just prior to using Loop vs. at 1 year of Loop use is illustrated in Fig. 3a-c. I have gotten really used to waking up with glucose levels I like the look of. Being able to deliver a bolus or set a temporary glucose target using my iPhone-not to mention my Apple Watch—still feels like something from the future!

It is impossible to say this pregnancy is going to be absolutely fine-many things could still happen-but so far achieving pregnancy glucose targets has been the least of my worries. That is entirely down to Loop and the support of my diabetes team. At each hospital appointment, I meet a medical student or someone from the wider diabetes and antenatal team who wants to learn about looping and how it works. With Tidepool poised to take Loop through the US Food and Drug Administration (FDA) and European regulatory processes, I hope many more people can have experiences and outcomes to date like mine in the future.

\section{PHYSICIAN PERSPECTIVE}

\section{What are Artificial Pancreas Systems?}

An artificial pancreas provides variable automated insulin doses in response to changes in interstitial glucose and other variables, some of which require manual input. It is the ultimate 
aim in providing optimal automated diabetes care and reducing burden for the person with T1D or their carers. Systems which come close to achieving this are being designed and run by a dedicated group of expert people managing T1D across an online "DIY" community. There are different names for these approaches including "looping" or "hybrid closed loops", DIY artificial pancreas systems (APS) or the more general term Automated Insulin Delivery Systems. These devices have evolved over recent years from early sensor-augmented pumps which decreased insulin dosing in response to hypoglycaemia (low-glucose suspend), to more advanced hybrid closed-loop systems [1]. Whilst these systems respond automatically to interstitial glucose, they still require user input for pre-meal bolus dosing, carbohydrate entry, glucose targets and optimisation of insulin profiles; therefore, they are hybrid closed-loop systems. In the future, fully closed-loop systems will become more common, where users do not have to enter meal boluses or carbohydrates, a scenario which has already arrived for some of the DIY APS user population.

\section{Why Would People with T1D Use These Devices?}

Outcomes in terms of improved glucose levels, reduced hypoglycaemic episodes, reduced variability, better overnight control and reduced burden are impressive. The systems also offer specific advantages in certain cases, as described in the stories above. Current estimates suggest that there are at least 1303 people using some form of DIY APS [2]. Advantages and disadvantages of DIY APS compared with conventional sensor-augmented therapy are summarized in Table 1.

Overall, DIY artificial pancreas approaches deliver tighter glycaemic control with increased time within target range and improved $\mathrm{HBA}_{1 \mathrm{c}}$ values compared with conventional therapies. James's $\mathrm{HbA}_{1 \mathrm{c}}$ reduced from 57 to $43 \mathrm{mmol} / \mathrm{L}$, with up to $90 \%$ of time in target range. Our other stories reflect similar improvements. This is consistent with clinical trials of hybrid closedloop systems [3, 4] but also evident in self- reported outcomes of DIY artificial pancreas systems [5-8]. What is also remarkable is the reduction in hypoglycaemia frequency or duration. Mendy describes how his toddler has on average one episode of nighttime hypoglycaemia per month, down from multiple incidents per week prior to looping.

The second common theme people with T1D report is an increase in quality of life [3]. DIY APS significantly reduce the time spent checking glucose readings, calculating insulin doses and reacting to peaks and troughs in glucose levels. For parents, carers and people with T1D, the reduced anxiety associated with fewer hypoglycaemic episodes is clearly invaluable.

There are also interesting benefits to people with T1D in more specific situations. Throughout pregnancy, both postmeal metabolism and insulin sensitivity change significantly. As illustrated in Melissa's example, DIY APS can help adapt to these insulin requirements and achieve tighter, safer glucose levels with less ongoing input from both the individual and healthcare professionals. Mendy clearly describes the difficulty in managing diabetes in a child with variable and unpredictable carbohydrate intake and activity; DIY APS can adapt dynamically and reduce carer anxiety.

At present, the Medtronic 670G is the only regulated and commercially available hybrid closed-loop automated insulin delivery system [9]. It has a fixed glucose target of $6.7 \mathrm{mmol} / \mathrm{L}$ (which can be temporarily raised to $8.3 \mathrm{mmol} / \mathrm{L}$ for exercise), and is not currently licensed for use in children aged under 7 or in pregnancy. This landmark technology is fairly conservative in its approach and lacks some of the flexibility and customisation options that DIY APS users tend to prioritise. Additional regulated automated insulin dosing devices, such as Diabeloop in France and Tidepool Loop in the USA, are anticipated to become commercially available soon; however, at present, DIY APS offer the most advanced and sophisticated way to manage glucose levels. Despite the significant initial investment of time to understand and set up these systems and their unregulated status, the advantages highlighted above, alongside strong DIY community support, provide compelling reasons for people with T1D to consider them. 
Table 1 Advantages and disadvantages for DIY APS as compared to conventional sensor-augmented pump therapy

\begin{tabular}{|c|c|}
\hline Advantages & Disadvantages \\
\hline $\begin{array}{l}\text { Reduced hypoglycaemic episodes, frequency and } \\
\text { duration }\end{array}$ & Unregulated and not medically approved \\
\hline Reduced glycaemic variability & Requires user engagement and activation \\
\hline Improved overnight glucose profile & Requires investment of time to understand and set up systems \\
\hline Reduced psychological burden and regimen burden & Requires a small additional financial investment \\
\hline $\begin{array}{l}\text { Improved time in range from automated insulin } \\
\text { delivery }\end{array}$ & $\begin{array}{l}\text { Technical support not available from HCP, clinics or device } \\
\text { manufacturers although available via DIY community }\end{array}$ \\
\hline Improved quality of life & $\begin{array}{l}\text { Technical proficiency needed although can be acquired and learnt via } \\
\text { DIY community support }\end{array}$ \\
\hline Helps adapt to changes in physiology & $\begin{array}{l}\text { Depending on algorithm used, compatible with certain but not all } \\
\text { pumps and CGM } \\
\text { Models of pumps that can be used for 'looping' are continuously } \\
\text { being expanded }\end{array}$ \\
\hline $\begin{array}{l}\text { Improved flexibility and customisation e.g. control } \\
\text { and connectivity to wearable devices }\end{array}$ & Increased battery usage of smartphone and pump \\
\hline \multicolumn{2}{|l|}{ Responsive DIY community support available } \\
\hline \multicolumn{2}{|l|}{$\begin{array}{l}\text { Algorithms and software continuously being } \\
\text { updated and improved }\end{array}$} \\
\hline \multicolumn{2}{|l|}{$\begin{array}{l}\text { Can overcome modest errors or miscalculations in } \\
\text { carb entries and bolus calculations }\end{array}$} \\
\hline $\begin{array}{l}\text { Allows remote monitoring (e.g. via Nightscout) for } \\
\text { carers }\end{array}$ & \\
\hline
\end{tabular}

\section{What is the Technical Setup?}

A DIY APS requires three connected components: a real-time CGM, a computer or smartphone with an algorithm for computing the insulin dose and an insulin pump [10] (Fig. 4). The three open-source systems currently available are OpenAPS, AndroidAPS and DIY Loop. Only specific insulin pumps are 'loopable'. Until recently loopers were using old Medtronic pumps (pre-2011), taking advantage of a radio frequency security protocol that allowed remote commands. Most recently, AndroidAPS developers have leveraged the Bluetooth protocols of newer pumps, and Insulet's OmniPod Eros pods are now also usable with DIY systems. Currently, all these systems are DIY and must be built by the user without clinician or manufacturer input. Significant online resources and responsive DIY community support facilitate setup and support ongoing use. Depending on the software a micro-computer (for OpenAPS) or smartphone (Android for AndroidAPS or iOS device for Loop) is required to run the algorithms and send commands to the pump. The systems can also allow integration and interoperability with other connected devices, such as smartwatches for checking system status and adjusting insulin delivery. 


\section{Automated diabetes:}

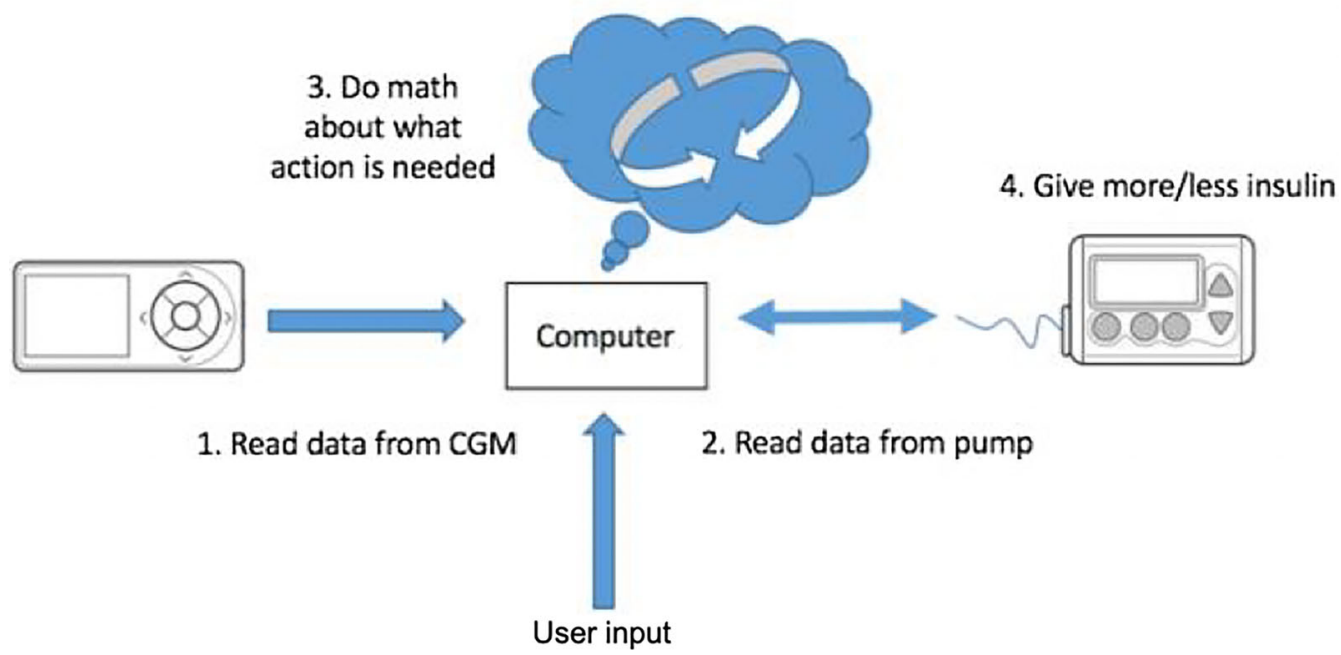

Fig. 4 Diagram of components required for hybrid closedloop automated insulin dosing systems such as DIY APS. Automated insulin dosing systems such as DIY APS require three connected components. These include realtime CGM to provide glucose data to a computer or smartphone with an algorithm for computing and doing the math needed for dosing insulin and a connected

\section{What is Healthcare Professional's Role?}

The use of DIY APS raises compelling ethical and medicolegal questions for healthcare professionals looking to support their use. These controversies are discussed in more detail by Barnard et al. [11]. A recent statement by the UK affiliate of JDRF (formerly known as the Juvenile Diabetes Research Foundation), the type 1 diabetes research charity, states that, although there are probable benefits, DIY APS cannot be endorsed and patients and carers use these at their own risk [12]. A similar statement from Diabetes Australia indicates the organisation also does not endorse this technology; however, if patients choose to use it then they should be supported by their healthcare team [13].

The precise ethical and legal framework in the UK is currently under discussion among professional bodies, the General Medical Council and medicolegal authorities. Initial feedback suggests that healthcare professionals (HCPs) are able to discuss this treatment if a

insulin pump to deliver insulin accordingly. User input of carbohydrate intake or any manual insulin adjustments are integrated by the computer or smartphone and influence the algorithm to alter insulin dosing (Figure adapted with permission from Lewis D, Automated Insulin Delivery, ISBN 9781797763699, https://www.artificialpancreasbook. com (C) Dana Lewis 2019)

person with diabetes asks them about it, but suggesting and recommending unregulated treatment options outside the context of a formal clinical trial would constitute off-label use. Supporting people with T1D who are currently using DIY APS requires balancing the risks and benefits for each individual, and clearly documenting discussions of the potential risks. A recent case also highlights the need for developing anonymised adverse event reporting systems for HCPs and potential role for regulatory bodies in facilitating this [14].

On the basis of our clinical experience, and as highlighted in the cases above, patients or carers who have a clear understanding of the risks, benefits, technical requirements and limitations of the automated insulin delivery systems, and who avail themselves of ongoing community support, can achieve much better metabolic outcomes with reduced psychological burden compared to traditional intensive insulin therapy [11]. Hence, in these circumstances, HCPs should continue to support people with 
T1D using DIY APS for items usually prescribed-including pump consumables, insulin, blood glucose and blood ketone monitoring and glucagon-and, where practicable, CGM, as it is in their best interests. We hope that forthcoming consensus statements will provide a clearer legal framework for HCPs to be able to do this, including supporting use by parents in children as in Mendy's example, during surgery as in James' example and in pregnancy as in Melissa's example.

\section{Complaince with Ethics Guidelines}

This article does not contain any studies with human or animal subjects performed by any of the authors

\section{SUMMARY}

In this report, three people managing T1D describe how they were able to set up DIY APS and how these devices have significantly improved their quality of life and ability to manage T1D. Experiences from use of these devices highlights impressive metabolic and psychological benefits. DIY APS approaches are not endorsed by regulatory bodies and the UK awaits clearer guidance on how clinicians can support people with T1D to use such unregulated systems. However, it is clear that we are in an exciting era where people with T1D are leading the way in moving artificial pancreas technology forward, reaping its benefits, supporting others to take advantage of it, and educating HCPs on its use. \#WeAreNotWaiting-are you?

\section{ACKNOWLEDGEMENTS}

The authors would like to thank Dana Lewis for her critical review and input.

Funding. No funding or sponsorship was received for this study or publication of this article. The authors are fully responsible for all content and editorial decisions and received no financial support or other form of compensation related to the development of this manuscript.

Authorship. All named authors meet the International Committee of Medical Journal Editors (ICMJE) criteria for authorship for this manuscript, take responsibility for the integrity of the work as a whole, and have given final approval for the version to be published.

Disclosures. Melissa Holloway has provided consulting services to Dexcom, Ascensia, Insulet, and Roche Diabetes Care. Anna Brackenridge declares non-promotional educational speaker honoraria from Abbott. Sufyan Hussain declares non-promotional educational speaker and advisory honoraria from Medtronic, Roche and Dexcom. Dominic C. Marshall, Mendy Korer and James Woodman have nothing to disclose.

Compliance with Ethics Guidelines. This article does not contain any studies with human or animal subjects performed by any of the authors.

Peer Review. Please note, contrary to the journal's standard single-blind peer review process, as a commentary this article underwent review by a member of the journal's Editorial Board.

Open Access. This article is distributed under the terms of the Creative Commons Attribution-NonCommercial 4.0 International License (http://creativecommons.org/licenses/ by-nc/4.0/), which permits any noncommercial use, distribution, and reproduction in any medium, provided you give appropriate credit to the original author(s) and the source, provide a link to the Creative Commons license, and indicate if changes were made.

\section{REFERENCES}

1. Kowalski A. Pathway to artificial pancreas systems revisited: moving downstream. Diabetes Care. 2015;38:1036 LP-1043. http://care.diabetesjournals. 
org/content/38/6/1036.abstract. Accessed 31 July 2019.

2. https://openaps.org/outcomes/. Accessed 31 July 2019.

3. Bekiari E, Kitsios K, Thabit H, et al. Artificial pancreas treatment for outpatients with type 1 diabetes: systematic review and meta-analysis. BMJ. 2018;361. https://www.bmj.com/content/361/bmj. k1310. Accessed 31 July 2019.

4. Choi SB, Hong E, Noh Y. Open artificial pancreas system reduced hypoglycemia and improved glycemic control in patients with type 1 diabetes. Diabetes. 2018;67. https://doi.org/10.2337/db18964-P. Accessed 31 July 2019.

5. Lewis D, Leibrand S, OpenAPS Community. Realworld use of open source artificial pancreas systems. J Diabetes Sci Technol. 2016;10(6):1411.

6. Litchman ML, Lewis D, Kelly LA, et al. Twitter analysis of \#OpenAPS DIY artificial pancreas technology use suggests improved A1C and quality of life. J Diabetes Sci Technol. 2019;13:164-70.

7. Lewis, DM, Swain, RS, Donner T. Improvements in A1C and time-in-range in DIY closed-loop (OpenAPS) users. Am Diabetes Assoc. 2018;67. https://doi. org/10.2337/db18-352-OR. Accessed 31 July 2019.

8. Provenzano V, Guastamacchia E, Brancato D, et al. Closing the loop with OpenAPS in people with type
1 diabetes-experience from Italy. Diabetes. 2018;67. https://doi.org/10.2337/db18-993-P. Accessed 31 July 2019.

9. Bergenstal RM, Garg S, Weinzimer SA, et al. Safety of a hybrid closed-loop insulin delivery system in patients with type 1 diabetes. JAMA. 2016;316:1407-8.

10. Hovorka R. Closed-loop insulin delivery: from bench to clinical practice. Nat Rev Endocrinol. 2011;7:385. https://doi.org/10.1038/nrendo.2011. 32. Accessed 31 July 2019.

11. Barnard KD, Ziegler R, Klonoff DC, et al. Open source closed-loop insulin delivery systems: a clash of cultures or merging of diverse approaches? J Diabetes Sci Technol. 2018;12:1223-6.

12. JDRF UK position statement on type 1 diabetes DIY technologies. https://jdrf.org.uk/about-us/positionstatements-reports/position-statements/jdrfs-uk-po sition-statement-on-type-1-diabetes-diy-technologi es/. Accessed 31 July 2019.

13. Diabetes Australia position statement: people with type 1 diabetes and do it yourself (DIY) technology solutions. https://www.diabetesaustralia.com.au/ position-statements. Accessed 31 July 2019.

14. FDA warns against use of unauthorized diabetes devices. Medscape Medical News. https://www. medscape.com/viewarticle/913195. Cited 23 June 2019. 